\title{
Prospects for direct neutron capture measurements on s-process branching point isotopes
} \author{
R. Reifarth ${ }^{5}$ \\ 1 Dpto. de Física Atómica, Molecular y Nuclear, Universidad de Sevilla, 41012 Sevilla, Spain \\ 2 Instituto de Física Corpuscular (CSIC-Universidad de Valencia), E-46071 Valencia, Spain \\ 3 Institut für Kernphysik, Karlsruhe Institute of Technology, 76021 Karlsruhe, Germany \\ 4 Dpto. de Ingeniería Electrónica, Universidad de Sevilla, 41012 Sevilla, Spain \\ ${ }^{5}$ Goethe-Universität Frankfurt am Main, 60323 Frankfurt am Main, Germany
}

C. Guerrero ${ }^{1, a}$, C. Domingo-Pardo ${ }^{2}$, F. Käppeler ${ }^{3}$, J. Lerendegui-Marco ${ }^{1}$, F.R. Palomo ${ }^{4}$, J.M. Quesada ${ }^{1}$, and

Received: 7 February 2017 / Revised: 14 March 2017

Published online: 10 May 2017

(c) The Author(s) 2017. This article is published with open access at Springerlink.com Communicated by C. Broggini

\begin{abstract}
The neutron capture cross sections of several unstable key isotopes acting as branching points in the $s$-process are crucial for stellar nucleosynthesis studies, but they are very challenging to measure directly due to the difficult production of sufficient sample material, the high activity of the resulting samples, and the actual $(n, \gamma)$ measurement, where high neutron fluxes and effective background rejection capabilities are required. At present there are about 21 relevant $s$-process branching point isotopes whose cross section could not be measured yet over the neutron energy range of interest for astrophysics. However, the situation is changing with some very recent developments and upcoming technologies. This work introduces three techniques that will change the current paradigm in the field: the use of $\gamma$-ray imaging techniques in $(n, \gamma)$ experiments, the production of moderated neutron beams using high-power lasers, and double capture experiments in Maxwellian neutron beams.
\end{abstract}

Nucleosynthesis of the elements heavier than iron is driven mainly by neutron capture reactions in stars. Half of the total amount of elements between $\mathrm{Cu}$ and $\mathrm{Bi}$ in the solar system was produced in the slow neutron capture process ( $s$-process) in He-burning layers of low-mass asymptotic giant branch (AGB) stars and during the Heand C-burning phases of massive stars. The corresponding nucleosynthesis studies involve detailed stellar modelling constrained by spectroscopic observations and meteoritic stardust grains in which reliable information on the nuclear physics part, in particular on the basic nuclear data such as half-lives and neutron capture (n, $\gamma$ ) cross sections, constitutes an essential ingredient $[1,2]$.

The most crucial and difficult data to obtain in this respect are the stellar neutron capture $(n, \gamma)$ cross sections of unstable isotopes, which are still very scarce or require substantial improvement. The importance of unstable isotopes is that they give rise to branchings in the $s$-process path due to the competition between $\beta$-decay and neutron capture, which depends on the neutron density, the Maxwellian Averaged Cross Section (MACS) and the mean thermal neutron velocity, i.e. the neutron energy

\footnotetext{
a e-mail: carlos.guerrero@cern.ch
}

distribution at a given temperature. In addition, both halflife and $(n, \gamma)$ cross section values depend in some cases on the temperature of the stellar site because of the population of some levels above the ground state. This dependence has a sizable effect on $s$-process abundance calculations for some isotopes [3] and cannot be measured, so measurements have to be combined with models $[4,5]$.

Since the isotopic abundance pattern within a branching is sensitive to the neutron density and temperature, knowledge of the neutron capture cross section of the involved unstable isotope provides very valuable information on these parameters at the corresponding stellar site. One such example is the measurement of the ${ }^{93} \mathrm{Zr}(\mathrm{n}, \gamma)$ cross section [6] which, in combination with spectroscopic observations and meteoritic stardust grains of extrinsic $\mathrm{S}$ stars made with HERMES [7], has been used to determine an upper-limit constraint of $2.5 \cdot 10^{8} \mathrm{~K}$ for the $s$-process temperature in low-mass AGB stars, a result which is independent of stellar evolution models [8]. Whereas the HERMES observational uncertainty on the $\mathrm{Zr} / \mathrm{Nb}$ abundance ratio is of only $3 \%$, the uncertainty contributed by the measured cross section is about $11 \%$. Clearly, a more accurate ${ }^{93} \mathrm{Zr}$ capture cross section, in particular extending the neutron energy limit from $8 \mathrm{keV}$ up to $30 \mathrm{keV}$, would 
help to improve the constraint on the $s$-process temperature considerably.

However, the instability of the branch-point isotopes represents a severe challenge for actual measurements due to at least one of the following reasons: i) these isotopes do not exist in nature and are difficult to produce in sizable quantities, ii) their half-life is too short (days or less) to allow a (weeks or months long) capture experiment, iii) their activity implies a considerable radiation hazard, and iv) their radiation represents an intense source of background that excludes in most cases standard measuring techniques used in currently available neutron beam facilities, being of no help to measure underground, as the prohibitive background comes from the sample itself. The list of branching point isotopes is long and includes more than 60 isotopes [9] whose half-life and $(\mathrm{n}, \gamma)$ cross section values have an impact on the abundance of a very large number of isotopes [10]. However, if one focuses on the 21 important $s$-process branching point isotopes identified in ref. [1] $\left({ }^{63} \mathrm{Ni},{ }^{79} \mathrm{Se},{ }^{81} \mathrm{Kr},{ }^{85} \mathrm{Kr},{ }^{95} \mathrm{Zr},{ }^{134,135} \mathrm{Cs},{ }^{147} \mathrm{Nd},{ }^{147,148} \mathrm{Pm}\right.$ ${ }^{151} \mathrm{Sm},{ }^{154,155} \mathrm{Eu},{ }^{153} \mathrm{Gd},{ }^{160} \mathrm{~Tb},{ }^{163} \mathrm{Ho},{ }^{170,171} \mathrm{Tm},{ }^{179} \mathrm{Ta}$, ${ }^{185} \mathrm{~W}$ and $\left.{ }^{204} \mathrm{Tl}\right)$, only ${ }^{63} \mathrm{Ni}\left(t_{1 / 2}=100 \mathrm{y}\right)$ [11-13] and ${ }^{151} \mathrm{Sm}\left(t_{1 / 2}=95 \mathrm{y}\right)[14]$ have been measured by time-offlight (ToF) so far, and just a few more by activation (see table III in ref. [1] for details). In the case of ${ }^{63} \mathrm{Ni}$, a sample with a ${ }^{63} \mathrm{Ni} /{ }^{62} \mathrm{Ni}$ ratio of 0.12 from extended reactor irradiations was available for $\sigma(\mathrm{n}, \gamma)$ measurements at $\mathrm{n}_{-}$TOFCERN [11,12] and DANCE-LANL [13]. These measurements represent a major improvement in constraining the high neutron fluence during convective shell C-burning in massive stars, prior to the supernova explosion.

Considering the above-mentioned challenges, there are still a few of the isotopes of interest that can be produced and measured using currently available techniques and facilities. For instance, a recent program was launched within the EU FP7 projects NEUTANDALUS and CHANDA for measuring by time-of-flight the capture cross section of the branching point isotopes ${ }^{147} \mathrm{Pm}$, ${ }^{171} \mathrm{Tm}$ and ${ }^{204} \mathrm{Tl}$. The samples have been produced by irradiating stable pellets of ${ }^{146} \mathrm{Nd},{ }^{170} \mathrm{Er}$ and ${ }^{203} \mathrm{Tl}$ at the ILL high flux reactor in France. The irradiated pellets were chemically purified at PSI in Switzerland and three samples suited for capture experiments were prepared. The cross section measurements at the $\mathrm{n}_{-}$TOF-EAR1 [15] and n_TOF-EAR2 [16] facilities have been completed in the summer of 2015 using the Total Energy Detection technique and the analysis is ongoing.

However, the challenges are too demanding for other isotopes. For instance, when measuring samples of the order of a mg the background often becomes dominant over the capture signal (especially in the unresolved resonance region) and thus experiments in the full energy range of interest can only be performed with new detection techniques that allow a better background suppression. In addition, the beam time needed when measuring mg samples becomes unaffordable at present ToF facilities. As an example, in the latest ${ }^{147} \mathrm{Pm}$ measurement performed at $\mathrm{n}_{-}$TOF-EAR2 (among the highest neutron flux facilities worldwide), a sample of $\sim 200 \mu \mathrm{g}$ was irradiated for more than 30 days and the statistics accumulated was only enough to identify a few of the low-lying/strongest resonances, which is not enough for extracting accurate MACS values. Last, even if sufficient material could be produced of isotopes such as ${ }^{95} \mathrm{Zr},{ }^{134} \mathrm{Cs},{ }^{147} \mathrm{Nd},{ }^{148} \mathrm{Pm}$, ${ }^{154} \mathrm{Eu},{ }^{160} \mathrm{~Tb},{ }^{170} \mathrm{Tm}$ or ${ }^{185} \mathrm{~W}$, the combination of their short half-lives and their high $\beta$-decay energies prevents their measurement with currently available techniques and facilities.

Based on the discussion above, a complete set of neutron energy differential capture cross sections for the branching point isotopes will only be achievable via new detection techniques for minimizing the background and new neutron beams with higher flux than currently available. For the cases where differential measurements are out of reach due to the combination of short half-life and high decay energy, double neutron capture experiments should be able to provide MACS values thanks to the availability of two new facilities featuring high continuous neutron fluxes with a Maxwellian spectrum. This paper contains the latest developments and plans regarding these three lines of action, including the experimental possibilities that each of them will open and the measurements that will become feasible upon the successful development of these ideas. While the focus is on radioactive isotopes, all the techniques described here can be applied as well to stable isotopes, especially in the cases where they are rare and thus available in very small quantities.

On this same topic, the reader is referred to ref. [17] for a recent original idea of using inverse kinematics combining a storage ring with radioactive ions passing through a dense neutron source, for instance a nuclear reactor. In addition, surrogate and surrogate ratio methods using for instance $(\gamma, \mathrm{n})[18]$ and $\left({ }^{18} \mathrm{O},{ }^{16} \mathrm{O}\right)[19]$ reactions, respectively, are currently being applied and can be of use for some of the unstable isotopes of interest. However, they have to rely largely on models for the unknown $\gamma$-ray transition probabilities, which limits the accuracy and applicability of these with respect to direct methods like the ones discussed herein.

Imaging applied to $(\mathrm{n}, \gamma)$ detectors. It has been mentioned already that a major challenge in $(n, \gamma)$ experiments on small samples is that the overall background dominates over the capture signals of interest. This is especially problematic for measuring and analysing heavy isotopes where the cross section in the energy range of astrophysical interest $(1-300 \mathrm{keV})$ is above the resonance region, making it very difficult to distinguish between capture and background events. In this regard, a leap forward is expected from the development of imaging Total Energy Detectors (i-TED) [20]. Using the principle of a Compton camera, the direction of origin of a detected photon can be determined from the energies and positions registered in a detector made of two position sensitive scintillators: the first one records the position where a Compton scattering takes place and the corresponding energy loss, and the second records a second position and the full energy of the scattered photon. A simple calculation using the Compton scattering formula provides a direction cone within which 


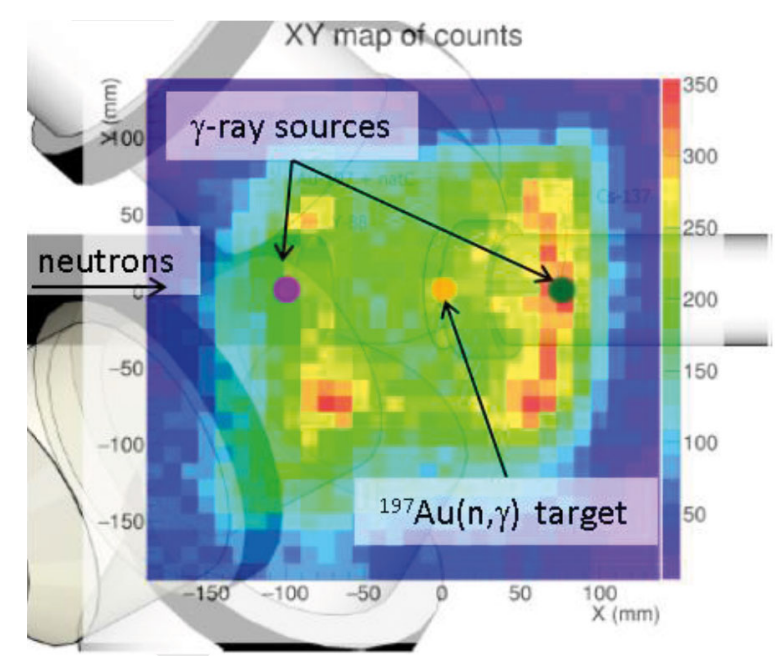

Fig. 1. Reconstructed origin of the photons during a ${ }^{197} \mathrm{Au}(\mathrm{n}, \gamma)$ experiment with $\gamma$-ray sources nearby the gold target. The $2 \mathrm{D}$ contour is superimposed on a sketch of the the four $\mathrm{C}_{6} \mathrm{D}_{6}$ detectors.

the photon was emitted. In this way, only those photons arriving from the direction of the sample position are considered in the analysis, therefore eliminating background coming from a different origin. This includes mainly neutron capture in the walls or the structural material by neutrons scattered at the sample or the vacuum windows, as well as the room background and the activity from the scintillators themselves (in the case of $\mathrm{LaBr}_{3}, \mathrm{BaF}_{2}$, etc.). Dedicated Geant4 [21] calculations using measured capture and background $\gamma$-ray spectra as input indicate that the capture to background ratio can be enhanced by an order of magnitude compared to presently used $\left(\mathrm{C}_{6} \mathrm{D}_{6}\right.$ detectors). The price of a reduced efficiency for an array of i-TED detectors is only a factor of four compared to the standard set-up of two $\mathrm{C}_{6} \mathrm{D}_{6}$ detectors.

In a first approach to the implementation of an optimized i-TED, a simplified set-up has been assembled, tested and characterized in terms of energy and spatial performance, both in the lab and at the $\mathrm{n}_{-} \mathrm{TOF}-\mathrm{EAR} 1$ neutron beam. It consists of a monolithic $\mathrm{CeBr}_{3}$ scintillating crystal coupled to a position-sensitive photomultiplier and an integrated circuit [22]. Using a lead pinhole collimator a series of dedicated measurements with calibrated sources and with a neutron beam incident on a ${ }^{197} \mathrm{Au}$ sample have been carried out at $\mathrm{n}_{-} \mathrm{TOF}$.

The imaging performance is illustrated in fig. 1 . It shows the reconstructed origin of photons in a ${ }^{197} \mathrm{Au}(\mathrm{n}, \gamma)$ experiment, where the background from additional $\gamma$-ray sources nearby the sample is strongly reduced. In spite of the very massive lead block placed near the detector, the signal-to-background ratio could be improved already by a factor of two selecting only $\gamma$-rays coming from the direction of the sample (see full details in ref. [23]). Based on this result, the work has started to design and build an optimized Compton camera to be tested at $\mathrm{n}_{-} \mathrm{TOF}$ during 2017.
Laser-driven neutron sources for $(\mathrm{n}, \gamma)$. While currently existing moderated neutron beams are based on particle accelerators, the use of high power (TW or even PW) fast-pulsed (ps or even fs) lasers is becoming a very promising option for particle acceleration, and thus for neutron production (see ref. [24] for a review of the current status of laser-driven neutron sources and the near future prospects). Among several methods and regimes for particle acceleration with laser and subsequent neutron production, the most widely employed is acceleration of light charged particles ( $p$ or $d$ ) in a pitcher by Target Normal Sheath Acceleration (TNSA) followed by the production of neutrons in a catcher via nuclear reactions such as p-Li, d-d, d-Be, etc. Using this technique the neutron production yields are of the order to $10^{6} \mathrm{n} / \mathrm{J}$. Accordingly, $2.5 \cdot 10^{8} \mathrm{n} / \mathrm{sr} /$ pulse have been produced at the Prague Asterix Laser System [25]. The record in terms of yield, however, has been achieved using the pitchercatcher scheme with the Break Out Afterburner (BOA) acceleration regime: in a recent experiment, Roth et al. [26] using the LANL 200 TW TRIDENT laser have produced a forward peaked neutron yield as high as $10^{10} \mathrm{n} / \mathrm{sr} /$ pulse of fast neutrons with energies depending on the emission angle and ranging between $100 \mathrm{keV}$ and $100 \mathrm{MeV}$, peaking between 3 and $10 \mathrm{MeV}$. Such an energy spectrum is not suited for stellar neutron capture measurements. However, if this initially fast neutron beam is moderated it could serve the energy range between 1 and $300 \mathrm{keV}$ of interest in astrophysics.

This has never been done before with a laser-driven neutron source, but dedicated calculations with Geant4 indicate that a ToF facility based on such a neutron source would provide an outstanding neutron beam in terms of flux and time/energy resolution in the neutron energy region of interest. The calculations presented herein are based on the neutron yield value obtained at LANL, a frequency of $10 \mathrm{~Hz}$ (a value that will be soon available at high-power lasers facilities, for instance at the European Extreme Light Infrastructure (ELI) [27], the use of a $10 \mathrm{~mm}$ water moderator, and a time-of-flight distance of $1 \mathrm{~m}$, similar to that of FRANZ [28]. Under these conditions, the corresponding neutron flux, displayed and compared to other ToF facilities in fig. 2, is higher in the $\mathrm{keV}$ region than all currently existing facilities, and comparable to the future FRANZ pulsed neutron beam.

Measuring $(\mathrm{n}, \gamma)$ reactions at such a short flight path would certainly be challenging, but both FRANZ and a laser-based facility of this type would be based on $\mathrm{MeV}$ light ion projectiles incident on thin and light targets $(\mathrm{Li}$ and Be in this case), thus using reactions other than spallation, in which high-energy $(\sim \mathrm{GeV})$ proton beams on heavy target (one ton of lead in the case of n_TOF) produce plenty of particles in addition to neutrons. The main problem for using $\gamma$-ray detectors in such environment will be the prompt flash of Bremsstrahlung radiation, from which the detectors will have to recover before the arrival of the neutrons, e.g. within $130 \mathrm{~ns}$ for $300 \mathrm{keV}$ neutrons.

If achieved, it would surely open a new range of possible experiments. If needed, the flight path could be increased and the reduction of neutron flux be partially 


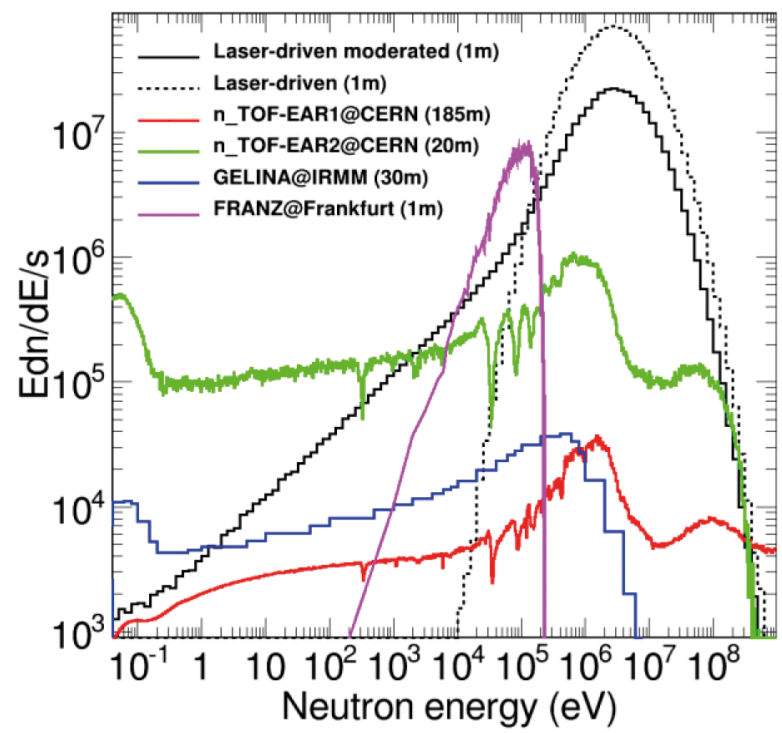

Fig. 2. Average neutron flux of a moderated laser-driven facility compared to that of the existing facilities $\mathrm{n}_{-} \mathrm{TOF}$ and GELINA, and the upcoming FRANZ.

compensated by the use of a thicker moderator, sacrificing part of the initially excellent time resolution of a laser-based facility. The optimization of these correlated parameters requires some input from experiments using a $\gamma$-ray detector near the laser-driven neutron source, which has not been done yet. This must be the first step towards a complete and realistic investigation of a possible laser-driven neutron time-of-flight facility. In addition, it remains to be investigated whether upcoming TW highrepetition table-top lasers could be used as portable white neutron sources, and thus open the possibility to combine the production of very short-lived nuclei with the neutron capture measurement at the production site, for instance at a research reactor.

Double neutron capture experiments. There are cases where differential (ToF) measurement are not feasible because the combination of the short half-life or the high energy decay of the radioactive isotope of interest are causing a background that is still too high for $\gamma$-ray detectors to stand. This has been seen recently in a ToF measurement of a ${ }^{171} \mathrm{Tm}$ sample containing just $\sim 16 \mu \mathrm{g}$ of ${ }^{170} \mathrm{Tm}$ $\left(t_{1 / 2}=128 \mathrm{~d}\right.$ and $\left.Q_{\beta}=0.97 \mathrm{MeV}\right)$ that made the gain and behaviour of the detectors very unstable and the handling of the sample very problematic.

In these cases, one could determine the integral $(\mathrm{n}, \gamma)$ cross section by exposing a sample ${ }^{A-1} Z$ to an appropriate neutron energy spectrum and inducing what is known as double captures. Provided that the neutron flux is high enough, the fraction of ${ }^{A+1} Z$ isotopes produced by double neutron captures ${ }^{A-1} Z(\mathrm{n}, \gamma)^{A} Z(\mathrm{n}, \gamma)^{A+1} Z$ (which is in competition with the decay of ${ }^{A} Z$ ) is sensitive to the unknown cross section of the unstable species ${ }^{A} Z$. Since the produced quantities of ${ }^{A+1} Z$ would be very small, this can only be applied to cases where this is unstable, otherwise the initial quantity of ${ }^{A+1} Z$ in the seed would be certainly

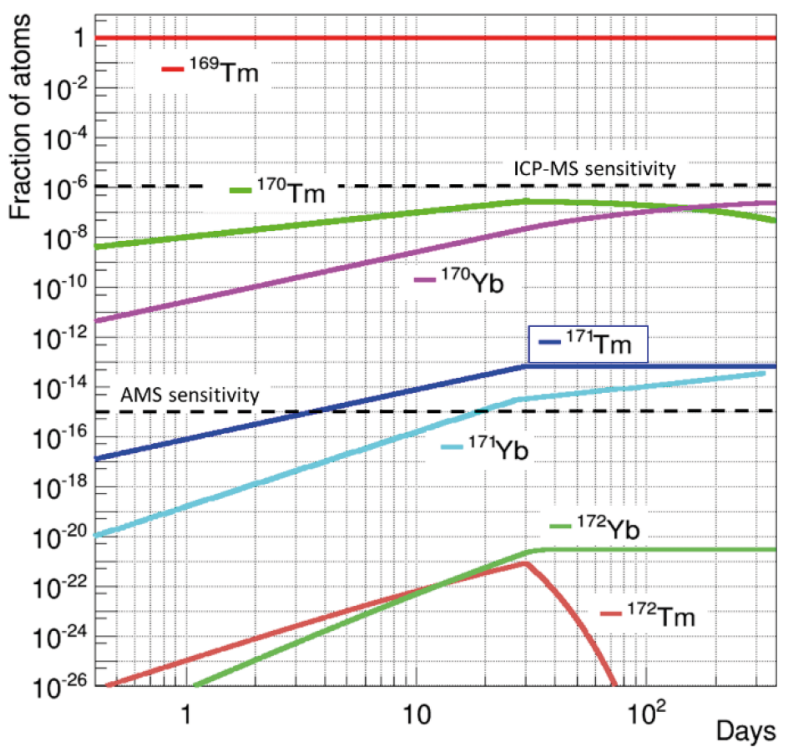

Fig. 3. Isotopic evolution during a year for a one-monthlong irradiation at FRANZ of fresh ${ }^{169} \mathrm{Tm}$ for determining the ${ }^{170} \mathrm{Tm}$ MACS value at $30 \mathrm{keV}$ via double capture.

larger than the one produced by double capture. These conditions apply to many important $s$-process branching point isotopes, for instance for ${ }^{59} \mathrm{Fe},{ }^{134} \mathrm{Cs},{ }^{135} \mathrm{Cs},{ }^{148} \mathrm{Pm}$, ${ }^{154} \mathrm{Eu},{ }^{155} \mathrm{Eu},{ }^{160} \mathrm{~Tb},{ }^{170} \mathrm{Tm},{ }^{171} \mathrm{Tm}$ and ${ }^{181} \mathrm{Hf}$. In the case of ${ }^{148} \mathrm{Pm}$ and ${ }^{171} \mathrm{Tm}$, the initial radioactive seeds would need to be produced ad hoc.

Once a sufficient fraction of ${ }^{A-1} Z$ are transformed into ${ }^{A+1} Z$ nuclei, one can determine the amount of ${ }^{A+1} Z$ produced from the corresponding activity, studying the change in $(n, \gamma)$ photons before and after the irradiation (see ref. [21] for the case of ${ }^{63} \mathrm{Ni}$ ), or determining the isotopic composition of the irradiated sample by mass spectroscopy. The most sensitive method is Accelerator Mass Spectroscopy (AMS), which allows one to measure isotopic ratios at the level of $10^{-15}$ [29], while inductively coupled-plasma mass spectroscopy (ICP-MS) allows reaching abundances down to $10^{-9}$ at best, but normally at the level of $10^{-6}$.

For providing useful values for astrophysics, these double capture experiments must be carried out using a neutron flux with a Maxwellian quasi-stellar neutron energy distribution. Until now, the intensities of such quasi-stellar neutron beams were too low for successful double neutron capture experiments, but this has changed with the existing LiLiT [30] and the upcoming FRANZ [28] facilities, which feature intensities of $10^{11} \mathrm{n} / \mathrm{s} / \mathrm{cm}^{2}$ with a Maxwellian neutron distribution of $30 \mathrm{keV}$. In order to illustrate the process, fig. 3 shows the calculated isotopic evolution of fresh ${ }^{169} \mathrm{Tm}$ irradiated with $10^{11} \mathrm{n} / \mathrm{s} / \mathrm{cm}^{2}$ for one month, using as input the MACS values from the KADoNiS database [31], and left to cool down for a year. It is observed how ${ }^{170} \mathrm{Tm}$ and ${ }^{171} \mathrm{Tm}$ are produced during the irradiation and how they decay continuously into ${ }^{170} \mathrm{Yb}$ and ${ }^{171} \mathrm{Yb}$ with half-lives of 128 days and 1.9 years, respectively. This shows that an irradiation at FRANZ or LiLiT would produce more than enough quantity (one hundred 
times larger than the AMS sensitivity) of ${ }^{171} \mathrm{Tm}$ via double capture, allowing to extract the MACS of ${ }^{170} \mathrm{Tm}$ and many others for the first time ever. One practical challenge in this and most double capture candidates is that the presence in the seed of isotopes other than ${ }^{A-1} Z$, even in very small quantities, may also produce ${ }^{A+1} Z$ nuclei (or isobars if mass spectroscopy is to be used) that would prevent the measurement. Therefore, the seed has to be physically and chemically purified before the irradiation. In the case of ${ }^{170} \mathrm{Tm}$, the commercially available material contains around $60 \mathrm{ppm}$ of ${ }^{170} \mathrm{Er}$ which, following neutron capture and $\beta$-decay, produces ${ }^{171} \mathrm{Tm}$ as well. Therefore the initial abundance of ${ }^{170} \mathrm{Er}$ must be minimized, in this case by combining chemical and mass separation, each of which would reduce the abundance of ${ }^{170} \mathrm{Er}$ by a factor $\sim 10^{-4}$, thus resulting in a final ${ }^{170} \mathrm{Er}$ abundance of $10^{-15}$, of which only $0.5 \%$ would transform into ${ }^{171} \mathrm{Tm}$, a negligible fraction compared to the $10^{-13}$ resulting from double capture on ${ }^{170} \mathrm{Tm}$.

The authors wish to thank U. Köster, D. Schumann and S. Heinitz for their valuable discussions and comments. This work and some of the mentioned experiments have been funded by the EC Marie Curie CIG Grant No. 334315 and the Spanish Ministerio de Economia y Competitividad under Grants No. FPA2011-24553 and FPA2013-45083-P.

Open Access This is an open access article distributed under the terms of the Creative Commons Attribution License (http://creativecommons.org/licenses/by/4.0), which permits unrestricted use, distribution, and reproduction in any medium, provided the original work is properly cited.

\section{References}

1. F. Käppeler, R. Gallino, S. Bisterzo, W. Aoki, Rev. Mod. Phys. 83, 157 (2011).

2. R. Reifarth, C. Lederer, F. Käppeler, J. Phys. G: Nucl. Part. Phys. 41, 053101 (2014).
3. J. Avila et al., Astrophys. J. 768, 1 (2013).

4. K. Cosner, J.W. Truran, Astrophys. Space Sci. 78, 85 (1981).

5. T. Rauscher, Astrophys. J. Lett. 755, 1 (2012).

6. G. Tagliente et al., Phys. Rev. C 87, 014622 (2013).

7. G. Raskin et al., Astron. Astrophys. 526, A69 (2011).

8. P. Neyskens, Nature, 517 (2015).

9. Astronomy with Radioactivities, edited by R. Diehl, D.H. Hartmann, Nikos Prantzos, Lect. Notes Phys., 812 (Springer, Berlin, 2011) pp. 83-152.

10. A. Koloczek et al., At. Data Nucl. Data Tables 108, 1 (2016).

11. C. Lederer et al., Phys. Rev. Lett. 110, 022501 (2013).

12. C. Lederer et al., Phys. Rev. C 89, 025810 (2014).

13. M. Weigand et al., Phys. Rev. C 92, 045810 (2015).

14. U. Abbondanno et al., Phys. Rev. Lett. 93, 161103 (2004).

15. C. Guerrero et al., Eur. Phys. J. A 49, 27 (2013).

16. C. Weiss et al., Nucl. Instrum. Methods A 799, 90 (2015).

17. R. Reifarth, Y.A. Litvinov, Phys. Rev. ST Accel. Beams 17, 014701 (2014).

18. R. Raut et al., Phys. Rev. Lett. 111, 112501 (2013).

19. S.Q. Yan et al., Phys. Rev. C 94, 015804 (2016).

20. C. Domingo-Pardo, Nucl. Instrum. Methods A 825, 78 (2016).

21. J. Allison et al., Nucl. Instrum. Methods A 835, 186 (2016).

22. V. Herrero-Bosch et al., IEEE Trans. Nucl. Sci. 58, 1641 (2011).

23. D.L. Pérez-Magán et al., Nucl. Instrum. Methods A $\mathbf{8 2 3}$, 107 (2016).

24. J. Alvarez et al., Phys. Procedia 60, 29 (2014).

25. D. Klir et al., Phys. Plasmas 22, 093117 (2015).

26. M. Roth et al., Phys. Rev. Lett. 110, 044802 (2013).

27. ELI-Extreme Light Infrastructure Science and Technology with Ultra-Intense Lasers Whitebook, edited by Gérard A. Mourou, Georg Korn, Wolfgang Sandner, John L. Collier (THOSS Media GmbH, 2011).

28. R. Reifarth et al., Publ. Astron. Soc. Aust. 26, 255 (2009) arXiv:0909.5279.

29. C. Tuniz, Radiat. Phys. Chem. 61, 317 (2001).

30. G. Feinberg et al., Nucl. Phys. A 827, 590c (2009).

31. I. Dillman et al., Nucl. Data Sheets 120, 171 (2014). 